\title{
Enzamin ameliorates adipose tissue inflammation with impaired adipocytokine expression and insulin resistance in $\mathrm{db} / \mathrm{db}$ mice
}

\author{
Yukinori Tamura ${ }^{1}$, Masato Yano ${ }^{1}$, Naoyuki Kawao ${ }^{1}$, Katsumi Okumoto ${ }^{2}$, Shigeru Ueshima ${ }^{3}$, Hiroshi Kaji ${ }^{1}$ \\ and Osamu Matsuo ${ }^{4} *$ \\ ${ }^{1}$ Department of Physiology and Regenerative Medicine, Kinki University Faculty of Medicine, Osakasayama, Japan \\ ${ }^{2}$ Life Science Research Institute, Kinki University, Osakasayama, Japan \\ ${ }^{3}$ Department of Food Science and Nutrition, Kinki University Faculty of Agriculture, Nara, Japan \\ ${ }^{4}$ Kinki University Faculty of Medicine, Osakasayama, Japan
}

(Received 2 May 2013 - Final revision received 29 September 2013 - Accepted 7 October 2013)

Journal of Nutritional Science (2013), vol. 2, e37, page 1 of 10

doi:10.1017/jns.2013.34

Abstract

The effects of Enzamin on obesity-related metabolic disorders in obese $\mathrm{db} / \mathrm{db}$ mice were examined to explore a novel agent for the prevention of insulin resistance. $\mathrm{Db} / \mathrm{db}$ mice were treated with water containing Enzamin $(0.1$ and $1.0 \%)$ for 8 weeks from 6 weeks of age. Enzamin treatment at $1.0 \%$, but not at $0.1 \%$, significantly decreased the fasting plasma glucose, serum total cholesterol and TAG levels in $\mathrm{db} / \mathrm{db}$ mice, without affecting body weight gain and body fat composition. Furthermore, insulin sensitivity and glucose tolerance were improved by the treatment of $\mathrm{db} / \mathrm{db}$ mice with $1.0 \%$ Enzamin. Immunohistochemical studies and gene expression analysis showed that $1.0 \%$ Enzamin treatment suppressed macrophage accumulation and inflammation in the adipose tissue. In addition, $1.0 \%$ Enzamin treatment increased serum adiponectin in $\mathrm{db} / \mathrm{db}$ mice. Treatment with $1.0 \%$ Enzamin also significantly suppressed the expression of NADPH oxidase subunits, suggesting an antioxidative effect for Enzamin in the adipose tissue. Furthermore, in vitro experiments demonstrated that the lipopolysaccharide-induced inflammatory reaction was significantly suppressed by Enzamin treatment in macrophages. Enzamin treatment increased the expression of GLUT4 mRNA in muscle, but not GLUT2 mRNA in the liver of db/db mice. Enzamin also increased the mRNA expression of carnitine palmitoyltransferase 1a (CPT1a, muscle isoform) in db/db mice, whereas Enzamin treatment did not affect the mRNA expression of CPT1b (liver isoform) in db/db mice. In conclusion, our data indicate that Enzamin can improve insulin resistance by ameliorating impaired adipocytokine expression, presumably through its anti-inflammatory action, and that Enzamin possesses a potential for preventing the metabolic syndrome.

Key words: Insulin resistance: Adipocytokines: Macrophages: Enzamin

The metabolic syndrome, which is characterised by a clustering of visceral obesity, impaired glucose tolerance, hypertension and dyslipidaemia, is a major cause of type 2 diabetes and atherothrombosis ${ }^{(1)}$. Visceral obesity and insulin resistance are thought to represent common underlying factors of the metabolic syndrome ${ }^{(2)}$. It is critical therefore to develop novel agents for the prevention or treatment of obesity and insulin resistance.
Many reports have indicated that obesity is associated with a state of chronic, low-grade inflammation, suggesting that inflammation may be a potential mechanism whereby obesity can lead to insulin resistance ${ }^{(3)}$. Indeed, obesity and insulin resistance are strongly associated with systemic markers of inflammation, and inflammation has been recognised clinically as a major predictor of atherosclerotic $\operatorname{diseases}^{(4)}$.

\footnotetext{
Abbreviations: ATCC, American Type Culture Collection; CPT, carnitine palmitoyltransferase; CPT1a, carnitine palmitoyltransferase 1 (liver); CPT1b, carnitine palmitoyltransferase 1 (muscle); CT, computed tomography; LPS, lipopolysaccharide; MCP-1, monocyte chemoattractant protein 1; Nox2, NADPH oxidase 2; PAI-1, plasminogen activator inhibitor 1 ; $\mathrm{t}-\mathrm{PA}$, tissue-type plasminogen activator.
}

* Corresponding author: Dr Osamu Matsuo, fax +8167635 9366, email matsuo-o@med.kindai.ac.jp 
The adipose tissue is an important endocrine organ that secretes numerous biologically active molecules, such as leptin, adiponectin, TNF- $\alpha$, monocyte chemoattractant protein 1 (MCP-1) and plasminogen activator inhibitor 1 (PAI-1), which are collectively termed adipocytokines ${ }^{(5,6)}$. The impaired production of pro-inflammatory and anti-inflammatory adipocytokines seen in visceral fat obesity is associated with the metabolic syndrome $e^{(7)}$, indicating that inflammatory changes in the adipose tissue may contribute to the development of several aspects of the metabolic syndrome and result in type 2 diabetes and thrombotic diseases ${ }^{(8)}$.

Enzamin is a product from Bacillus subtilis $\mathrm{AK}$ and Lactobacillus, treated under physically adverse conditions such as heat treatment, and acid treatment ${ }^{(9)}$. We have shown previously that Enzamin displays profibrinolytic anti-thrombotic properties both in vitro and in vivo ${ }^{(9)}$. However, the effects of Enzamin on the metabolic syndrome have not yet been elucidated. In the present study, we therefore examined the influence of Enzamin on insulin resistance and adipocytokine expression in obese diabetic $\mathrm{db} / \mathrm{db}$ mice.

\section{Experimental methods}

\section{Materials}

The following materials were obtained from the commercial sources indicated: human regular insulin (Eli Lilly), anti-F4/ 80 antibody (AbD Serotec), Glutest Ace (Sanwa Kagaku Kenkyusho), RNeasy mini kits (Qiagen), rat monoclonal anti-F4/80 antibody (AbD Serotec), anti-rat secondary antibodies conjugated with horseradish peroxidase (Cell Signaling Technology Japan), fetal bovine serum (American Type Culture Collection (ATCC)) and lipopolysaccharides (LPS) from Escherichia coli 0111:B4 (Sigma Aldrich Japan). All other reagents and chemicals were of the highest grade available. Enzamin, which was produced by the long-term fermentation and self-digestion of Bacillus subtilis $\mathrm{AK}$ and Lactobacillus $^{(10)}$, was provided by the Enzamin Research Institute, Osaka, Japan. Japan Food Research Laboratories, which issue the official documents for the food export, analysed the molecular distribution of Enzamin by HPLC with a TSKgel G2500PWXL column (TOSOH Corporation). The standard molecular markers were cytochrome $c(12500 \mathrm{Da})$, aprotinin $(6152 \mathrm{Da})$, bacitracin $(1450 \mathrm{Da})$, angiotensin II (1046 Da), Gly-Gly-Tyr-Arg (451 Da) and Gly-Gly-Gly $(189 \mathrm{Da})$. There was an abundance of peaks and the majority were below the molecular weight of $500 \mathrm{Da}(75 \%)$. There were peaks with the molecular weight of $500-1000$ Da (10\%), 1000-3000 Da (11\%), 3000-6000 Da (2\%) and over $6000 \mathrm{Da}(2 \%)$, respectively.

\section{Animal preparation and experimental design}

Male $\mathrm{db} / \mathrm{db}$ mice and $\mathrm{db} /+\mathrm{m}$ mice were obtained from Charles River at 5 weeks of age. The $\mathrm{db} / \mathrm{db}$ mice were fed on normal chow and water ad libitum supplemented with 0 , 0.1 or $1.0 \%$ Enzamin for 8 weeks from 6 weeks of age. Each $0 \%$ Enzamin group is referred to below as the control.
The $\mathrm{db} /+\mathrm{m}$ mice were fed on normal chow and water ad libitum as lean mice. All animals were maintained on a $12 \mathrm{~h}$ lightdark cycle. The animal experiments were conducted according to the Guidelines for Animal Experiments at Kinki University Faculty of Medicine.

\section{Body fat composition analysis}

Body fat composition was estimated by computed tomography (CT) analysis in mice that were anaesthetised with forane (Isoflurane; Abbott Japan) and then scanned using a LaTheta (LCT-200) experimental animal CT system (Hitachi-Aloka Medical) $^{(11)}$. Contiguous 1-mm slice images between L1 and L5 were used for quantitative assessment employing LaTheta software (version 3.40). Visceral fat and subcutaneous fat were distinguished, and the total fat content, visceral fat weight and subcutaneous fat weight were calculated from all slice images and evaluated quantitatively.

\section{Analysis of metabolic parameters}

The plasma insulin, serum total cholesterol, TAG, adiponectin and TNF- $\alpha$ levels were measured using an insulin assay kit (Morinaga Institute of Biological Science), Cholesterol E test, Triglyceride E test (Wako Pure Chemical Industries), adiponectin ELISA kit (Otsuka Pharmaceutical) and a Quantikine TNF- $\alpha$ ELISA kit (R\&D Systems), respectively. For glucose tolerance tests, mice were deprived of food for $16 \mathrm{~h}$ and glucose (1.5 g/kg body mass) was then injected intraperitoneally. For insulin tolerance tests, mice were injected intraperitoneally with human regular insulin $(10.5 \mu \mathrm{g} / \mathrm{kg}$ body mass for $\mathrm{db} /+$ $\mathrm{m}$ mice and $35.0 \mu \mathrm{g} / \mathrm{kg}$ body mass for $\mathrm{db} / \mathrm{db}$ mice). Blood samples were collected before and after each injection, and the plasma glucose concentration was measured with a Glutest Ace.

\section{Cell culture}

RAW 264.7 (ATCC number: TIB-71), a murine macrophage cell line, was purchased from ATCC and maintained in Dulbecco's modified essential medium supplemented with $10 \%$ fetal bovine serum and $100 \mathrm{mg} / \mathrm{ml}$ penicillin-streptomycin at $37^{\circ} \mathrm{C}$ with $5 \% \mathrm{CO}_{2}$. RAW 264.7 cells $\left(1 \times 10^{6}\right.$ cells/well) were plated on six-well cell culture plates and incubated for $24 \mathrm{~h}$. Cells were treated with 0.01 or $0.1 \%$ concentrations of Enzamin in the presence or absence of $1 \mu \mathrm{g} / \mathrm{ml}$ LPS for $12 \mathrm{~h}$. After washing twice with PBS, the total cellular RNA was extracted for gene expression analysis employing real-time PCR.

\section{Quantitative real-time PCR}

Total RNA was extracted from the frozen adipose tissue (100 $\mathrm{mg})$, liver tissue $(30 \mathrm{mg})$ and muscle tissue $(30 \mathrm{mg})$ of mice and from RAW 264.7 cells using an RNeasy mini kit (Qiagen). The cDNA was synthesised from the total RNA using Super Script III (Life Technologies Japan). The real-time PCR was performed on a StepOne Plus using the SYBR ${ }^{\circledR}$ Green PCR Master Mix (Life Technologies Japan). The primer 
Table 1. Primers used for real-time PCR

\begin{tabular}{|c|c|c|}
\hline Primer & & Sequence \\
\hline \multirow[t]{2}{*}{ GLUT2 } & Forward & 5'-GGCTAATTTCAGGACTGGTT-3' \\
\hline & Reverse & 5'-TTTCTTTGCCCTGACTTCCT-3' \\
\hline \multirow[t]{2}{*}{ GLUT4 } & Forward & 5'-CATGGCTGTCGCTGGTTTC-3' \\
\hline & Reverse & 5'-AAACCCATGCCGACAATGA-3' \\
\hline \multirow[t]{2}{*}{ G6Pase } & Forward & 5'-CTGTGAGACCGGACCAGGA-3' \\
\hline & Reverse & 5'-GACCATAACATATACACCTGCTGC-3' \\
\hline \multirow[t]{2}{*}{ CPT1a } & Forward & 5'-GTCCCAGCTGTCAAAGATAC-3' \\
\hline & Reverse & 5'-GGAAGTATTGAAGAGTCGC-3' \\
\hline \multirow[t]{2}{*}{ CPT1b } & Forward & 5'-CAAGTTCAGAGACGAACGCC-3' \\
\hline & Reverse & 5'-TCAAGAGCTGTTCTCCGAACTG-3' \\
\hline \multirow[t]{2}{*}{ TNF- $\alpha$} & Forward & 5'-CCCAGACCCTCACACTCAGATC-3' \\
\hline & Reverse & 5'-GCCACTCCAGCTGСТССТC-3' \\
\hline \multirow[t]{2}{*}{ MCP-1 } & Forward & 5'-ССАСТСАССТGСТGСТАСТСА-3' \\
\hline & Reverse & 5'-TGGTGATCCTCTTGTAGCTCTCC-3' \\
\hline \multirow[t]{2}{*}{ IL-6 } & Forward & 5'-GTTCTCTGGGAAATCGTGGA-3' \\
\hline & Reverse & 5'-GGAAATTCGGGGTAGGAAGGA-3' \\
\hline \multirow[t]{2}{*}{ PAI-1 } & Forward & 5'-TTCAGCCCTTGCTTGCCTC-3' \\
\hline & Reverse & 5'-ACACTTTTACTCCGAAGTCGGT-3' \\
\hline \multirow[t]{2}{*}{ Emr-1 } & Forward & 5'-CTTTGGCTATGGGCTTCCAGTC-3' \\
\hline & Reverse & 5'-GCAAGGAGGACAGAGTTTATCGTG-3' \\
\hline \multirow[t]{2}{*}{ CD68 } & Forward & 5'-CTTCCCACAGGCAGCACAG-3' \\
\hline & Reverse & 5'-AATGATGAGAGGCAGCAAGAGG-3' \\
\hline \multirow[t]{2}{*}{ TLR-4 } & Forward & 5'-TATCCAGGTGTGAAATTGAAACAATT-3' \\
\hline & Reverse & 5'-GGGTTTCCTGTCAGTATCAAGTTTG-3' \\
\hline \multirow[t]{2}{*}{ Nox2 } & Forward & 5'-TTGGGTCAGCACTGGCTCTG-3' \\
\hline & Reverse & 5'-TGGCGGTGTGCAGTGCTATC-3' \\
\hline \multirow[t]{2}{*}{ P22 $2^{\text {phox }}$} & Forward & 5'-GTCCACCATGGAGCGATGTG-3' \\
\hline & Reverse & 5'-CAATGGCCAAGCAGACGGTC-3' \\
\hline \multirow{2}{*}{$\mathrm{P} 47^{\text {phox }}$} & Forward & 5'-GATGTTCCCCATTGAGGCCG-3' \\
\hline & Reverse & 5'-GTTTCAGGTCATCAGGCCGC-3' \\
\hline \multirow[t]{2}{*}{ 18S rRNA } & Forward & 5'-CGGCTACCACATCCAAGGAA-3' \\
\hline & Reverse & 5'-GCTGGAATTACCGCGGCT-3' \\
\hline
\end{tabular}

G6Pase, glucose-6-phosphatase; CPT1a, carnitine palmitoyltransferase 1 (liver); CPT1b, carnitine palmitoyltransferase 1 (muscle); MCP-1, monocyte chemoattractant protein 1; PAI-1, plasminogen activator inhibitor 1; Emr-1, EGF-like module-containing mucin-like hormone receptor-like 1; TLR-4, Toll-like receptor 4; Nox2, NADPH oxidase 2; rRNA, ribosomal RNA.

sets are listed in Table 1. The mRNA levels were normalised relative to the amount of $18 \mathrm{~s}$ ribosomal RNA, and expressed in arbitrary units.

\section{Histological analysis}

Adipose tissue was fixed for $12-16 \mathrm{~h}$ at $4^{\circ} \mathrm{C}$ with $4 \%$ paraformaldehyde, and embedded in paraffin. Sections of $4 \mu \mathrm{m}$ thickness were incubated with rat monoclonal anti-F4/80 antibody. The sections were then incubated with the appropriate anti-rat secondary antibodies conjugated with horseradish peroxidase. Positive signals were visualised employing a tyramide signal amplification system (PerkinElmer). The sections were counterstained with 4',6-diamidino-2-phenylindole (DAPI) and photographed using a fluorescence microscope (E800; Canon) with a CCD camera (Keyence Japan). For each individual mouse tissue block, ten fields from each section were analysed. The total number of nuclei and the number of nuclei of F4/80-expressing cells were counted for each field by employing an image processing program (NIH image; National Institutes of Health) in a blinded evaluation. The fraction of F4/80-expressing cells for each section was calculated as the sum of the number of nuclei of F4/80-expressing cells divided by the total number of nuclei in the sections.

\section{Statistical analysis}

All data were expressed as mean values with their standard errors. The statistical significance of differences was assessed by the unpaired $t$ test and one-way ANOVA. Differences with $P<0.05$ were regarded as significant. All statistical analyses were performed using StatView version 5.0 software (SAS Institute Inc.).

\section{Results}

\section{Effect of Enzamin on body fat composition in $d b / d b$ mice}

Treatment with $1 \%$ Enzamin exerted no effect on water intake, whereas treatment with $0 \cdot 1 \%$ Enzamin increased water intake slightly. Enzamin had no effect on food intake (data not shown) or body weight gain in $\mathrm{db} / \mathrm{db}$ mice (Fig. 1 (a)). To examine the effect of Enzamin treatment on body fat composition, we next performed CT scan analysis. Abdominal CT images at L2 demonstrated that both the visceral and subcutaneous fat areas were markedly increased in $\mathrm{db} / \mathrm{db}$ mice as compared with $\mathrm{db} /+\mathrm{m}$ mice, but that Enzamin treatment exerted no effect on the visceral and subcutaneous fat areas in $\mathrm{db} / \mathrm{db}$ mice (Fig. 1(b)). The total fat content, visceral fat weight and subcutaneous fat weight calculated from the CT scan analysis were not affected by Enzamin 
(a)
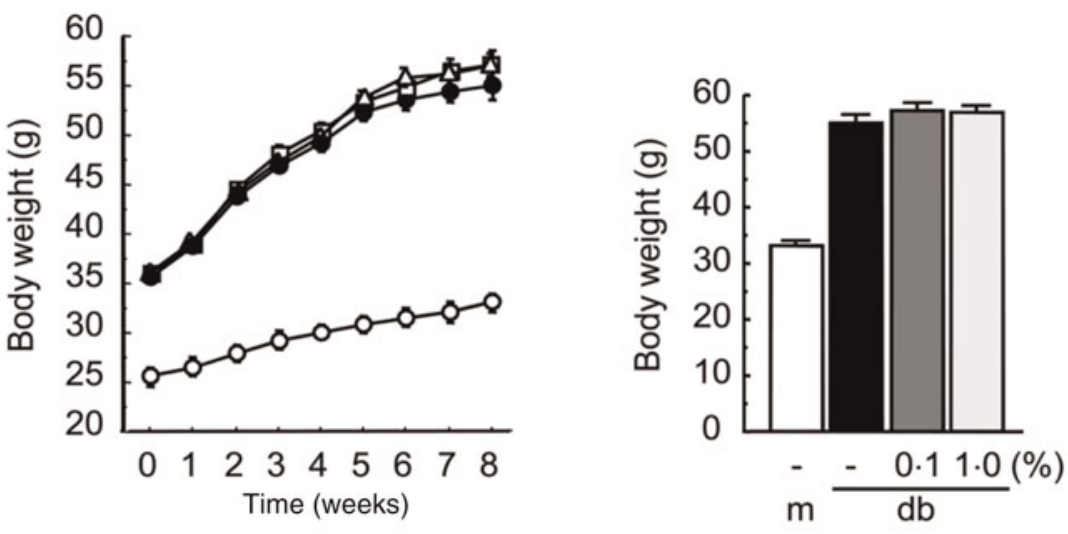

(b)

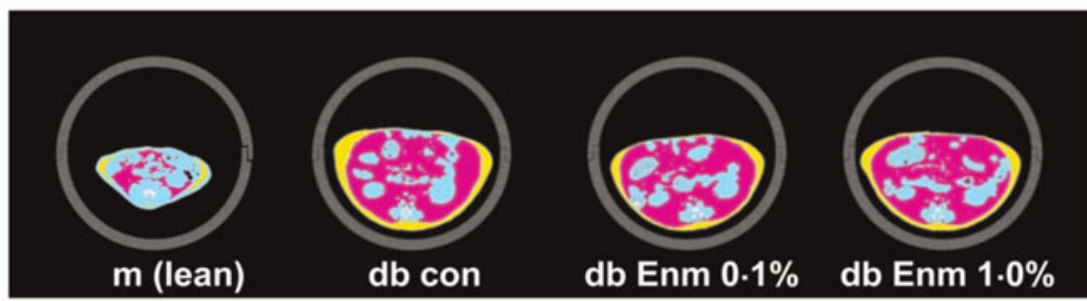

(c)

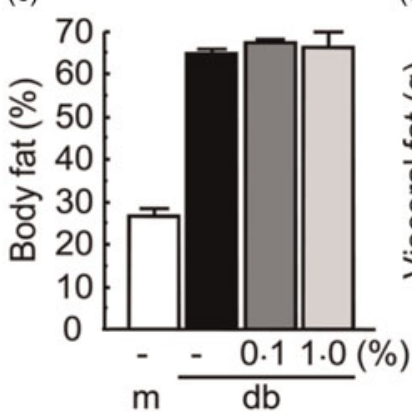

(d)

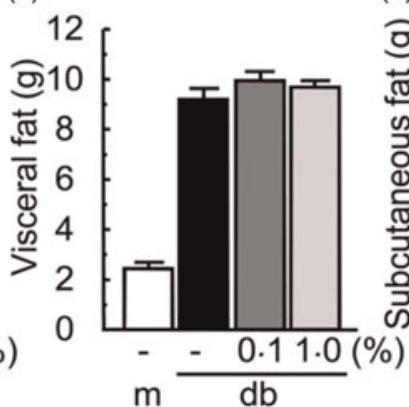

(e)

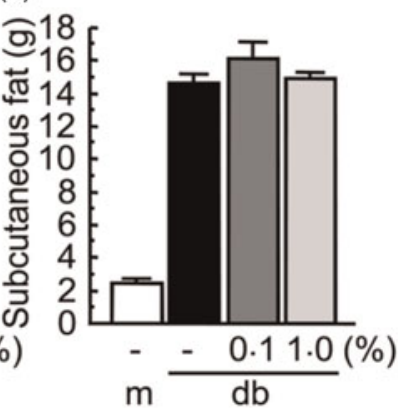

Fig. 1. Effect of Enzamin (Enm) treatment for 8 weeks on body weight and fat composition. (a) Growth curves during the experiment (left) and body weight at the end of the experiment (right) of non-treated db/ $+\mathrm{m}$ (lean control) and db/db (obese control) mice, and 0.1 and $1.0 \%$ Enzamin-treated db/db mice. $\bigcirc$, $\mathrm{db} /+\mathrm{m} \mathrm{mice}(n 6)$; - non-treated db/db mice ( $n$ 10); $\Delta, 0.1 \%$ Enzamin-treated db/db mice ( $n 9$ ); $\square, 1.0 \%$ Enzamin-treated db/db mice ( $n$ 8). (b) Representative computed tomography (CT) sections of abdominal regions. con, Control. Pink areas show visceral fat; yellow areas show subcutaneous fat. (c) Total fat content, and weights of visceral (d) and subcutaneous (e) fat in $\mathrm{db} /+\mathrm{m}$ and $\mathrm{db} / \mathrm{db}$ mice following each treatment, calculated from CT scan data. m, db/ $+\mathrm{m}$; db, db/db. Data are means ( $n$ 6-10 per group), with standard errors represented by vertical bars.

treatment in $\mathrm{db} / \mathrm{db}$ mice (Fig. 1(c)-(e)). Enzamin treatment also had no effect on the liver fat content estimated by CT scan analysis in $\mathrm{db} / \mathrm{db}$ mice (data not shown).

\section{Effect of Enzamin on lipid and glucose metabolism in $\mathrm{db} / \mathrm{db}$ mice}

To evaluate the effect of Enzamin treatment on lipid metabolism, we measured serum TAG and total cholesterol levels in $\mathrm{db} / \mathrm{db}$ mice. The fasting serum TAG levels in $\mathrm{db} / \mathrm{db}$ mice were higher than those in $\mathrm{db} / \mathrm{m}$ mice (Fig. 2(a)). Treatment with Enzamin at $0.1 \%$ slightly reduced the serum TAG level, and 1.0\% Enzamin treatment significantly reduced the fasting serum TAG level in db/db mice (Fig. 2(a)). Similarly, the fasting serum total cholesterol level was slightly but not significantly decreased by $0.1 \%$ Enzamin, and was significantly decreased by $1.0 \%$ Enzamin in $\mathrm{db} / \mathrm{db}$ mice (Fig. 2(b)). Thus, Enzamin treatment reduced fasting serum TAG and cholesterol levels dose-dependently in $\mathrm{db} / \mathrm{db}$ mice. Taken together, these findings suggest that Enzamin treatment improves lipid metabolism in $\mathrm{db} / \mathrm{db}$ mice.

We next evaluated the effect of Enzamin treatment on glucose metabolism in $\mathrm{db} / \mathrm{db}$ mice. The fasting plasma glucose was markedly increased in $\mathrm{db} / \mathrm{db}$ mice as compared with $\mathrm{db} /+\mathrm{m}$ mice (Fig. 2(c)). Enzamin treatment at $1.0 \%$, but not at $0.1 \%$, significantly reduced the fasting plasma glucose level in $\mathrm{db} / \mathrm{db}$ mice (Fig. 2(c)). Although the fasting plasma insulin was also markedly elevated in $\mathrm{db} / \mathrm{db}$ mice as compared with $\mathrm{db} /+\mathrm{m}$ mice (Fig. 2(d)), there were no differences in fasting plasma insulin levels between non-treated $\mathrm{db} / \mathrm{db}$ mice and Enzamin-treated db/db mice (Fig. 2(d)).

To evaluate further the effects of Enzamin on glucose metabolism, we next performed an intraperitoneal glucose tolerance test and intraperitoneal insulin tolerance test. The plasma glucose level after intraperitoneal glucose injection was dramatically elevated in $\mathrm{db} / \mathrm{db}$ mice as compared with $\mathrm{db} /+\mathrm{m}$ mice (Fig. 3(a)), indicating severe glucose intolerance. Enzamin treatment at $1.0 \%$, but not at $0.1 \%$, significantly 
(a)
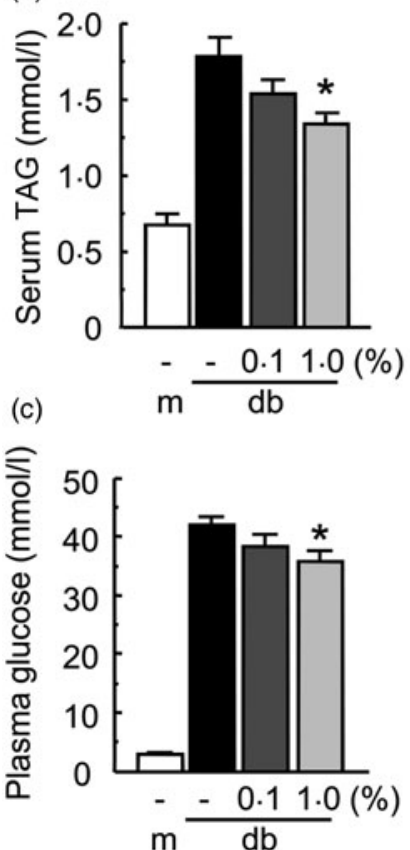

(b)
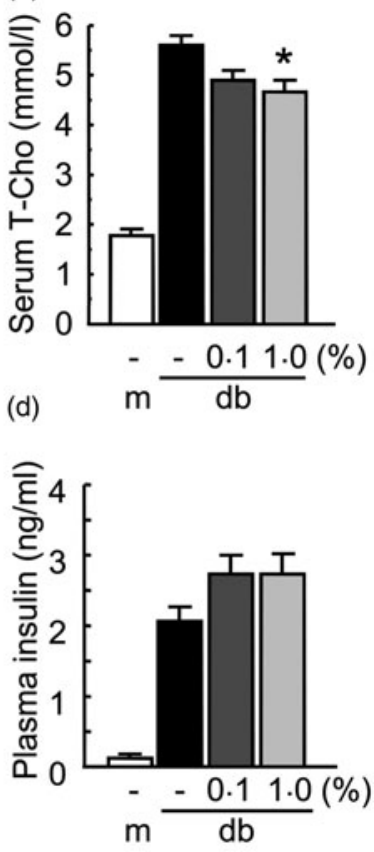

Fig. 2. Effect of Enzamin treatment for 8 weeks on lipid and glucose metabolism. Fasting serum TAG (a), total cholesterol (T-Cho) (b), plasma glucose (c) and insulin (d) concentrations in non-treated $\mathrm{db} /+\mathrm{m}$ (lean control, $n$ 6) and $\mathrm{db} / \mathrm{db}$ (obese control, $n$ 10) mice, and $0.1 \%(n 9)$ and $1.0 \%(n 8)$ Enzamin-treated db/db mice. $\mathrm{m}, \mathrm{db} / \mathrm{m} ; \mathrm{db}, \mathrm{db} / \mathrm{db}$. Data are means $(n$ 6-10 per group), with standard errors represented by vertical bars. *Mean value was significantly different from that of the non-treated $\mathrm{db} / \mathrm{db}$ mice $(P<0.05)$.

suppressed the elevation of the plasma glucose level in $\mathrm{db} / \mathrm{db}$ mice at 30 min after glucose injection. Furthermore, a similar suppressive effect was also noted at 60 and 90 min after intraperitoneal glucose injection (Fig. 3(a)). These data suggest that Enzamin can improve glucose intolerance in $\mathrm{db} / \mathrm{db}$ mice. Severe impairment of insulin sensitivity was also observed in $\mathrm{db} / \mathrm{db}$ mice as compared with $\mathrm{db} /+\mathrm{m}$ mice (Fig. 3(b)). Although $0.1 \%$ Enzamin treatment exerted no effect on insulin sensitivity in $\mathrm{db} / \mathrm{db}$ mice, $1.0 \%$ Enzamin treatment significantly decreased the plasma glucose level in response to insulin at 30, 60, 90 and $120 \mathrm{~min}$ after intraperitoneal insulin injection in $\mathrm{db} / \mathrm{db}$ mice (Fig. 3(b)). Taken together, these data suggest

that Enzamin treatment can ameliorate insulin resistance in $\mathrm{db} / \mathrm{db}$ mice.

We next evaluated the gene expression of GLUT2 (the main GLUT in liver) and glucose-6-phosphatase (G6Pase, an enzyme related to gluconeogenesis) in the liver, and the gene expression of GLUT4 (a muscular GLUT) in the muscle of $\mathrm{db} / \mathrm{db}$ mice. Enzamin treatment did not affect the mRNA expression of GLUT2 and G6Pase in the liver of $\mathrm{db} / \mathrm{db}$ mice (Fig. 4(a) and (b)), suggesting that Enzamin does not affect glucose metabolism in the liver of $\mathrm{db} / \mathrm{db}$ mice. The mRNA expression of GLUT4 in muscle was markedly decreased in $\mathrm{db} / \mathrm{db}$ mice as compared with $\mathrm{db} /+\mathrm{m}$ mice (Fig. 4(d)). Enzamin treatment at $1.0 \%$ significantly increased the mRNA expression of GLUT4 in the muscle of $\mathrm{db} / \mathrm{db}$ mice as compared with non-treated $\mathrm{db} / \mathrm{db}$ mice (Fig. 4(d)). We evaluated the gene expression of carnitine palmitoyltransferase 1 (CPT1, a mitochondrial enzyme related with lipid oxidation) in the liver and muscle of $\mathrm{db} / \mathrm{db}$ mice. The mRNA expression of CPT1a (liver isoform) and CPT1b (muscle isoform) was significantly decreased in $\mathrm{db} / \mathrm{db}$ mice as compared with $\mathrm{db} /+\mathrm{m}$ mice (Fig. 4(c) and (e)). Enzamin treatment at $1.0 \%$ increased the mRNA expression of CPT1b in the muscle of $\mathrm{db} / \mathrm{db}$ mice as compared with non-treated $\mathrm{db} / \mathrm{db}$ mice (Fig. 4(e)), whereas Enzamin treatment did not affect the mRNA expression of CPT1a in the liver of $\mathrm{db} / \mathrm{db}$ mice (Fig. 4(c)). These data suggest that Enzamin treatment improves glucose uptake and lipid oxidation in the muscle of $\mathrm{db} / \mathrm{db}$ mice.

\section{Effect of Enzamin on adipocytokine expression in $d b / d b$ mice}

To clarify the mechanism whereby Enzamin improves insulin resistance, we examined the effect of Enzamin treatment on pro-inflammatory adipocytokine expression in the adipose tissue of $\mathrm{db} / \mathrm{db}$ mice. The mRNA expression of TNF- $\alpha$ in the adipose tissue of $\mathrm{db} / \mathrm{db}$ mice was 5 -fold higher than that in $\mathrm{db} /+\mathrm{m}$ mice, indicating adipose tissue inflammation in $\mathrm{db} / \mathrm{db}$ mice (Fig. 5(a)). Enzamin treatment suppressed the TNF- $\alpha$ mRNA expression dose-dependently in the adipose tissue of $\mathrm{db} / \mathrm{db}$ mice. In particular, $1.0 \%$ Enzamin treatment significantly suppressed TNF- $\alpha$ mRNA expression by $40 \%$ in the adipose tissue of $\mathrm{db} / \mathrm{db}$ mice (Fig. 5(a)). Consistent with
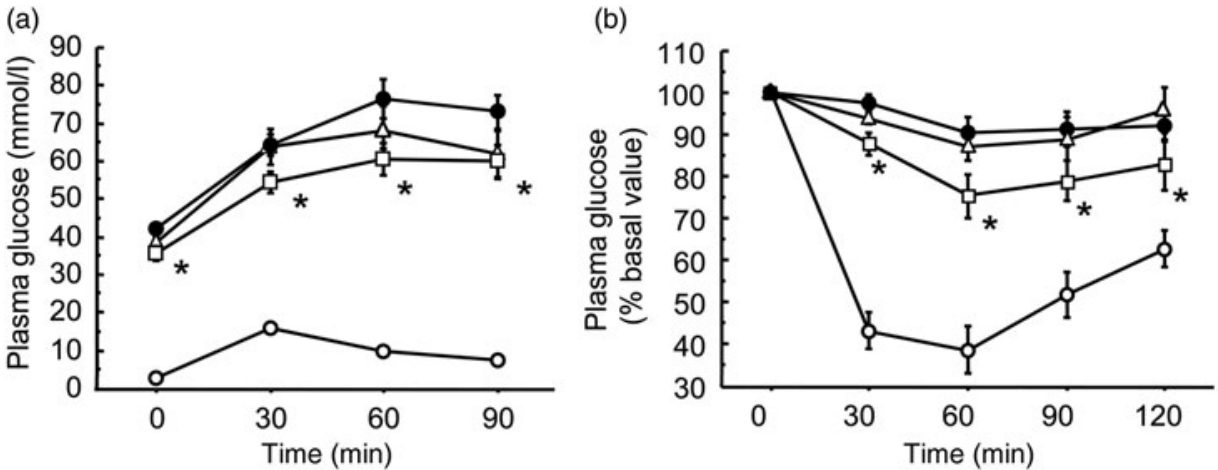

Fig. 3. Effects of Enzamin treatment for 8 weeks on glucose tolerance and insulin sensitivity. Responses of plasma glucose to a single intraperitoneal injection of glucose (a) or insulin (b) in non-treated db/ $+\mathrm{m}(\bigcirc, n 6)$ and db/db $(\bullet, n 10)$ mice, and $0.1 \%(\Delta, n 9)$ and $1.0 \%(\square, n 8)$ Enzamin-treated db/db mice. m, db/ + m; db, $\mathrm{db} / \mathrm{db}$. Data are means ( $n$ 6-10 per group), with standard errors represented by vertical bars. *Mean value was significantly different from that of the non-treated $\mathrm{db} / \mathrm{db}$ mice $(P<0.05)$. 

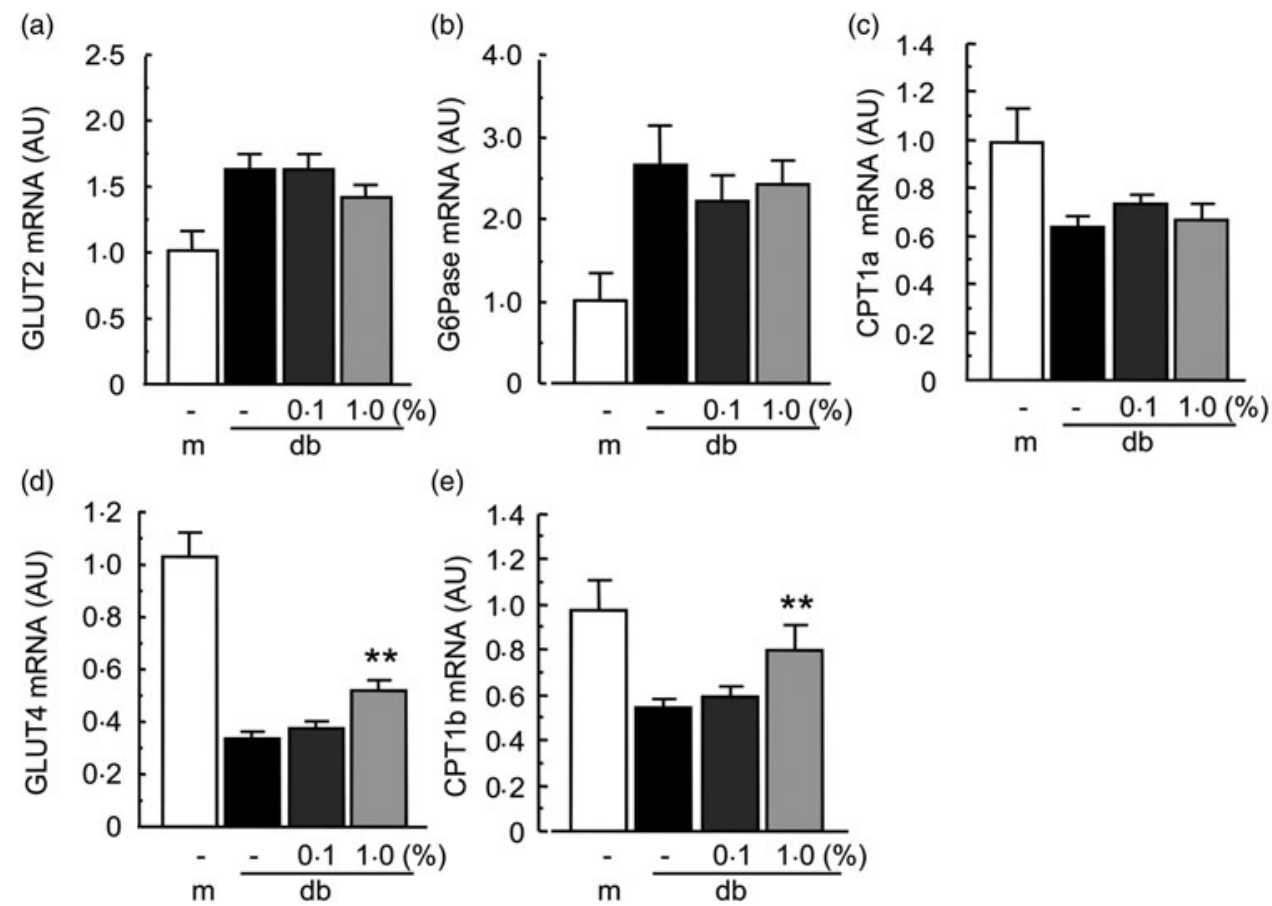

Fig. 4. Effect of Enzamin treatment for 8 weeks on glucose and lipid metabolism in liver and muscle. Messenger RNA (mRNA) expression of GLUT2 (a), glucose-6phosphatase (G6Pase) (b) and carnitine palmitoyltransferase (CPT) 1a (c) in the liver, and GLUT4 (d) and CPT1b (e) in the muscle of non-treated db/ $+\mathrm{m}$ (lean control, $n 6$ ) and db/db (obese control, $n$ 10) mice, and $0.1 \%(n 9)$ and $1.0 \%(n 8)$ Enzamin-treated db/db mice. AU, arbitrary units; m, db/ + m; db, db/db. Data are means ( $n$ 6-10 per group), with standard errors represented by vertical bars. ${ }^{* *}$ Mean value was significantly different from that of the non-treated $\mathrm{db} / \mathrm{db}$ mice $(P<0.01)$.
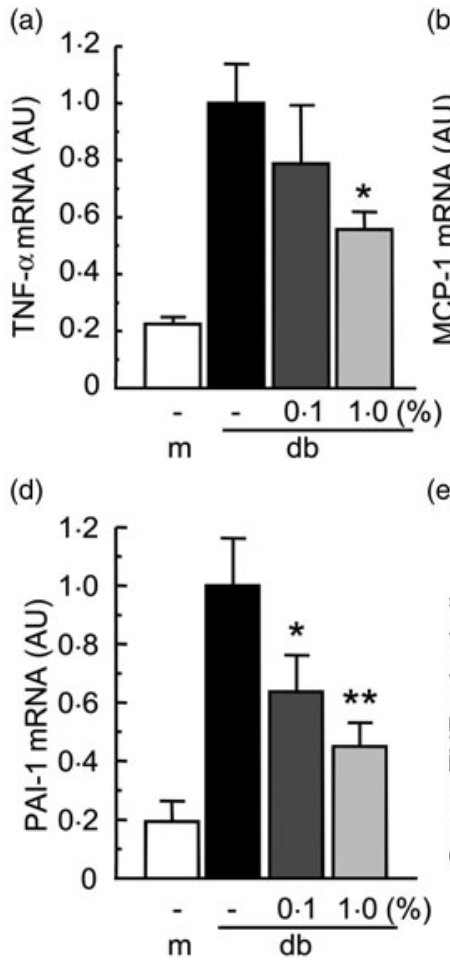

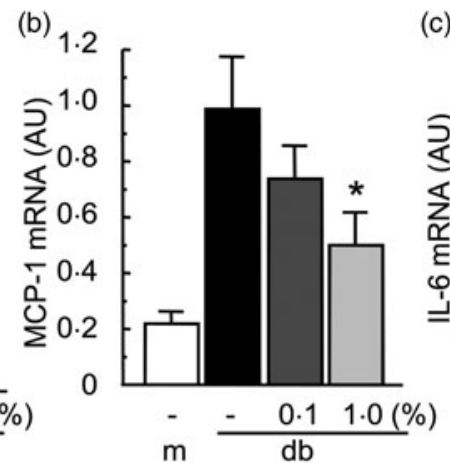

(e)

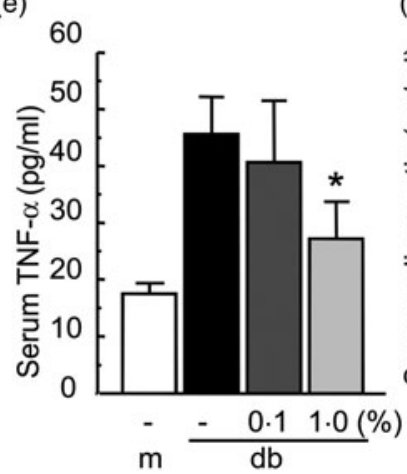

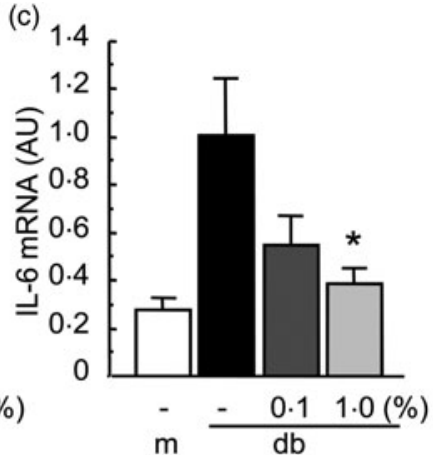

(f)

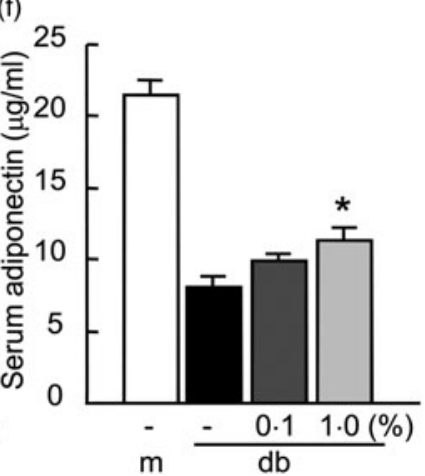

Fig. 5. Effect of Enzamin treatment for 8 weeks on adipocytokine expression. Messenger RNA (mRNA) expressions of TNF- $\alpha$ (a), monocyte chemoattractant protein 1 (MCP-1) (b), IL-6 (c) and plasminogen activator inhibitor 1 (PAI-1) (d) in epididymal white adipose tissue, and serum concentrations of TNF- $\alpha$ (e) and adiponectin ( $f$ ) in non-treated db/ + m (lean control, $n 6$ ) and db/db (obese control, $n 10)$ mice, and $0.1 \%(n 9)$ and $1.0 \%(n 8)$ Enzamin-treated db/db mice. AU, arbitrary units; m, db/ $+\mathrm{m} ; \mathrm{db}, \mathrm{db} / \mathrm{db}$. Data are means ( $n$ 6-10 per group), with standard errors represented by vertical bars. Mean value was significantly different from that of the nontreated $\mathrm{db} / \mathrm{db}$ mice: ${ }^{\star} P<0.05,{ }^{\star \star} P<0.01$. 

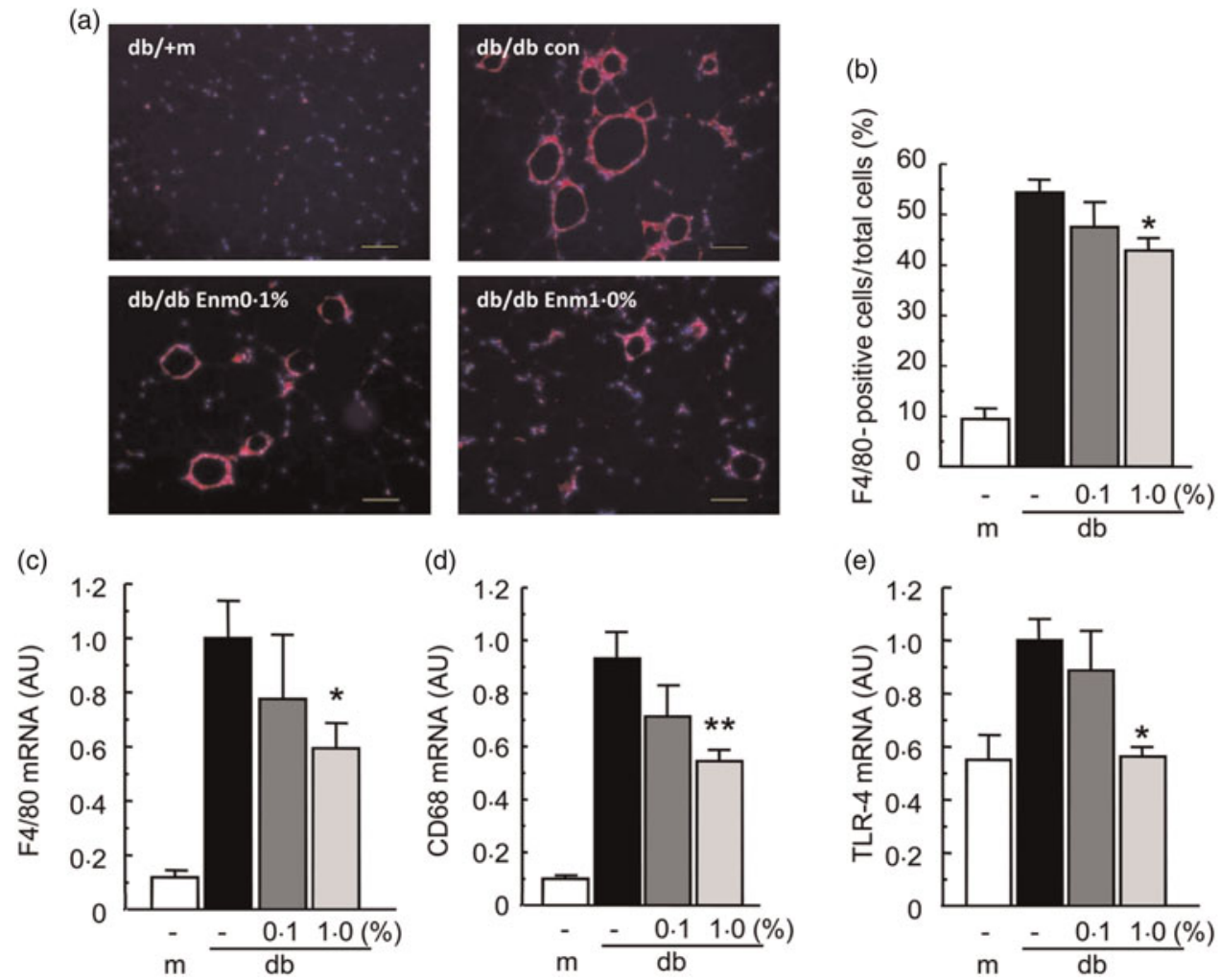

Fig. 6. Effect of Enzamin (Enm) treatment for 8 weeks on macrophage accumulation in adipose tissue. (a) Macrophage content of epididymal white adipose tissue (WAT) as assessed by F4/80 staining (bar, $100 \mu \mathrm{m}$ ) and (b) fraction of adipose tissue macrophages (F4/80-stained cells/total cells) in epididymal WAT of non-treated $\mathrm{db} /+\mathrm{m}$ (lean control) and db/db (obese control) mice, and $0.1 \%$ and $1.0 \%$ Enzamin-treated db/db mice ( $n 5$ for each group). con, Control. Messenger RNA (mRNA) expressions of EGF-like module-containing mucin-like hormone receptor-like 1 (Emr-1) (c), CD68 (d) and Toll-like receptor 4 (TLR-4) (e) in adipose tissue of nontreated db/ + m (lean control, $n 6$ ) and db/db (obese control, $n 10)$ mice, and $0.1 \%(n 9)$ and $1.0 \%$ ( $n$ 8) Enzamin-treated db/db mice. AU, arbitrary units; m, db/ + m; $\mathrm{db}, \mathrm{db} / \mathrm{db}$. Data are means, with standard errors represented by vertical bars. Mean value was significantly different from that of the non-treated $\mathrm{db} / \mathrm{db}$ mice: ${ }^{\star} P<0.05$, ${ }^{\star *} P<0.01$.

this, $1.0 \%$ Enzamin also suppressed the mRNA expression of MCP-1 and IL-6 by 50 and $60 \%$, respectively, in the adipose tissue of $\mathrm{db} / \mathrm{db}$ mice (Fig. 5(b) and (c)). Furthermore, the PAI-1 mRNA level was significantly reduced by both $0 \cdot 1$ and $1.0 \%$ Enzamin in the adipose tissue of $\mathrm{db} / \mathrm{db}$ mice (Fig. 5(d)). These data suggest that Enzamin treatment can suppress adipose tissue inflammation in $\mathrm{db} / \mathrm{db}$ mice.

To evaluate the effect of Enzamin on systemic inflammation, we measured the serum TNF- $\alpha$ level in $\mathrm{db} / \mathrm{db}$ mice. Enzamin treatment at $1.0 \%$, but not at $0.1 \%$, significantly suppressed the circulating TNF- $\alpha$ level in $\mathrm{db} / \mathrm{db}$ mice (Fig. 5(e)), suggesting that Enzamin could suppress systemic inflammation as well as adipose tissue inflammation in $\mathrm{db} / \mathrm{db}$ mice. In contrast to pro-inflammatory adipocytokines, the level of serum adiponectin was significantly increased by treatment with Enzamin in a dose-dependent manner. In particular, $1.0 \%$ Enzamin treatment increased serum adiponectin by 1.4 -fold in $\mathrm{db} / \mathrm{db}$ mice (Fig. 5(f)). These data suggest that Enzamin can improve the impaired adipocytokine expression in obese mice.

\section{Effect of Enzamin on macrophage accumulation and activation in $\mathrm{db} / \mathrm{db}$ mice}

To evaluate the effect of Enzamin on macrophage accumulation in adipose tissue, we performed immunohistochemical staining for F4/80, a mature macrophage marker, in adipose tissue sections from obese mice. The histological photographs indicated that macrophage accumulation was dramatically increased in $\mathrm{db} / \mathrm{db}$ mice as compared with $\mathrm{db} /+\mathrm{m}$ mice, and Enzamin treatment appeared to reduce dose-dependently the F4/80-positive area in the adipose tissue of $\mathrm{db} / \mathrm{db}$ mice (Fig. 6(a)). Consistent with the histological photographs, the fraction of F4/80-positive cells in the adipose tissue was slightly, but not significantly, suppressed by $0.1 \%$ Enzamin, and significantly suppressed by $1.0 \%$ Enzamin treatment in db/db mice (Fig. 6(b)). Furthermore, 1.0 \% Enzamin treatment significantly decreased the mRNA expression of EGF-like module-containing mucin-like hormone receptor-like 1 (Emr-1; an F4/80 antigen) in the adipose tissue of $\mathrm{db} / \mathrm{db}$ mice by $40 \%$ (Fig. 6(c)). In addition, the mRNA expressions of CD68 and Toll-like receptor 4 (TLR-4), a marker of activated and pro-inflammatory macrophages, were decreased by Enzamin treatment in the adipose tissue of $\mathrm{db} / \mathrm{db}$ mice (Figs. 6(d) and (e)). These data indicate that Enzamin may suppress pro-inflammatory macrophage accumulation and activation in the adipose tissue of $\mathrm{db} / \mathrm{db}$ mice.

Effect of Enzamin on oxidative stress in adipose tissue of $\mathrm{db} /$ db mice

To evaluate the effect of Enzamin on oxidative stress in adipose tissue, we measured the expression of NADPH oxidase 
(a)

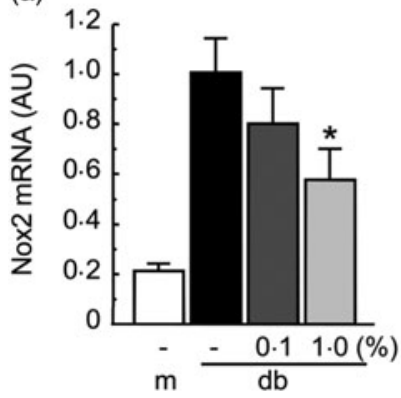

(b)

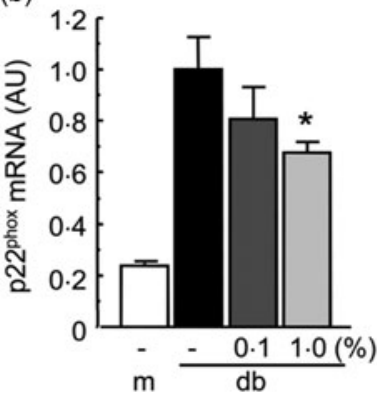

(c)

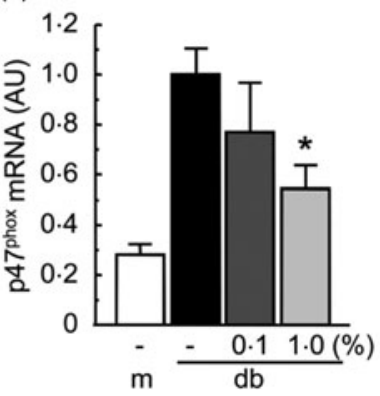

Fig. 7. Effect of Enzamin treatment for 8 weeks on oxidative stress in adipose tissue. Messenger RNA (mRNA) expressions of NADPH oxidase (Nox) subunits, Nox2 (a), p22 ${ }^{\text {phox }}$ (b) and p47 $7^{\text {phox }}$ (c) in adipose tissue of non-treated db/ $+\mathrm{m}$ (lean control, $\left.n 6\right)$ and db/db (obese control, $\left.n 10\right)$ mice, and $0.1 \%(n$ ) and $1.0 \%(n 8)$ Enzamin-treated $\mathrm{db} / \mathrm{db}$ mice. AU, arbitrary units; $\mathrm{m}, \mathrm{db} / \mathrm{m} ; \mathrm{db}, \mathrm{db} / \mathrm{db}$. Data are means ( $n$ 6-10 per group), with standard errors represented by vertical bars. ${ }^{*}$ Mean value was significantly different from that of the non-treated $\mathrm{db} / \mathrm{db}$ mice $(P<0.05)$.

subunits in adipose tissue from $\mathrm{db} / \mathrm{db}$ mice. The expressions of NADPH oxidase subunits, such as NADPH oxidase 2 (Nox2), $\mathrm{p} 22^{\text {phox }}$ and $\mathrm{p} 47^{\text {phox }}$, in non-treated $\mathrm{db} / \mathrm{db}$ mice were markedly higher than those in $\mathrm{db} /+\mathrm{m}$ mice (Fig. 7 (a)-(c)). Although there was no significant effect, $0 \cdot 1 \%$ Enzamin treatment tended to suppress the expressions of $\mathrm{NADPH}$ oxidase subunits. Furthermore, treatment with $1.0 \%$ Enzamin significantly suppressed the mRNA expressions of Nox $2, \mathrm{p} 22^{\text {phox }}$ and $\mathrm{p} 47^{\text {phox }}$ by 40,30 and $40 \%$, respectively, in the adipose tissue of db/db mice (Fig. 7(a)-(c)). These data suggest that Enzamin treatment can suppress oxidative stress in the adipose tissue of obese mice.

\section{Effect of Enzamin on lipopolysaccharide-induced inflammatory response in macrophages in vitro}

To evaluate the influence of Enzamin on the inflammatory response in macrophages, we investigated the effect of Enzamin on LPS-induced TNF- $\alpha$ expression in RAW 264.7 cells, a murine macrophage cell line. Although Enzamin treatment slightly increased the mRNA expression of TNF- $\alpha$ in macrophages without LPS stimulation, the LPS-induced elevation of TNF- $\alpha$ expression was significantly suppressed

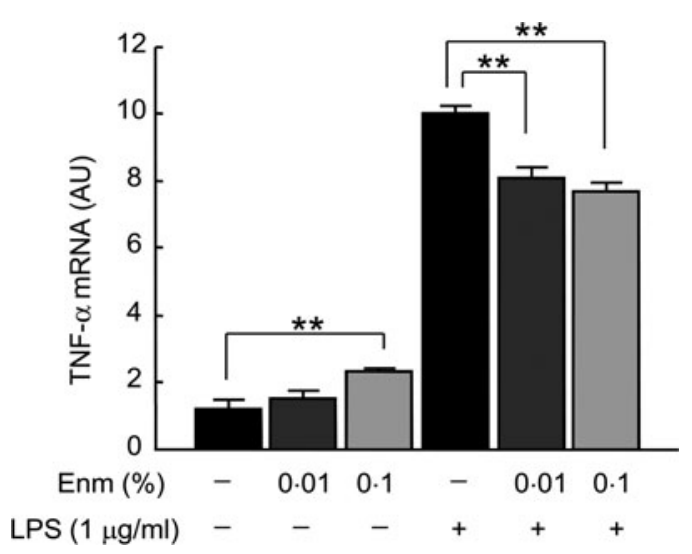

Fig. 8. Effect of Enzamin (Enm) treatment on lipopolysaccharide (LPS)-induced inflammatory response in macrophage in vitro. Messenger RNA (mRNA) expressions of TNF- $\alpha$ in RAW 264.7 cells treated without or with Enzamin $(0.01$ and $0.1 \%)$ for $12 \mathrm{~h}$, in the absence or presence of LPS $(1 \mu \mathrm{g} / \mathrm{ml})$. AU, arbitrary units. Data are means ( $n 3$ per group), with standard errors represented by vertical bars. ${ }^{* *} P<0.01$. by both the 0.01 and $0.1 \%$ Enzamin treatments (Fig. 8), suggesting that Enzamin tends to suppress the pro-inflammatory response in macrophages.

\section{Discussion}

In the present study, we demonstrated that Enzamin can improve the insulin resistance and impaired adipocytokine expression in $\mathrm{db} / \mathrm{db}$ mice. We found that Enzamin treatment suppressed macrophage accumulation in the adipose tissue as well as adipose tissue inflammation in $\mathrm{db} / \mathrm{db}$ mice. Several studies have shown that the expression of pro-inflammatory cytokines by adipose tissue is in part attributable to the expression of these cytokines by non-adipocytes, including macrophages ${ }^{(12,13)}$. Furthermore, Suganami et al. ${ }^{(14,15)}$ developed an in vitro co-culture system composed of adipocytes and macrophages, and demonstrated that a paracrine loop involving NEFA and TNF- $\alpha$ derived from the adipocytes and macrophages, respectively, leads to a vicious cycle that augments the inflammatory changes in both adipocytes and macrophages, i.e. marked up-regulation of pro-inflammatory cytokines such as MCP-1 and TNF- $\alpha$ and down-regulation of the anti-inflammatory cytokine adiponectin. Moreover, in a previous study, we found that pharmacological inhibition of macrophage infiltration suppressed adipose tissue inflammation and improved the impairment of adipocytokine production $^{(16)}$. In the present experiments, therefore, Enzamin might have suppressed adipose tissue inflammation by inhibiting macrophage infiltration into the adipose tissue of obese mice.

Many reports have suggested that adipose tissue inflammatory changes contribute to the development of insulin resistance. It has been demonstrated that adipose tissue-derived pro-inflammatory cytokines such as TNF- $\alpha^{(17,18)}$, and IL- $6^{(19,20)}$ can actually cause insulin resistance in experimental models. In the present study, Enzamin treatment at 1.0 \% reduced the serum TNF- $\alpha$ level in $\mathrm{db} / \mathrm{db}$ mice, suggesting that Enzamin can suppress systemic inflammation in $\mathrm{db} / \mathrm{db}$ mice. Furthermore, TNF- $\alpha$ dose-dependently reduces the expression of adiponectin in adipocytes by suppressing its promoter activity ${ }^{(21,22)}$. Adiponectin serves as an insulinsensitising agent ${ }^{(6,23)}$, so that a decrease in plasma adiponectin is related to insulin resistance in obesity. In the present study, 
we observed that the serum adiponectin level was decreased in $\mathrm{db} / \mathrm{db}$ mice as compared with $\mathrm{db} /+\mathrm{m}$ mice, in addition to an impaired insulin sensitivity and glucose tolerance. However, Enzamin treatment improved hypoadiponectinaemia in $\mathrm{db} /$ $\mathrm{db}$ mice. The increase in adiponectin by Enzamin treatment therefore may be responsible for the improvement of insulin resistance in $\mathrm{db} / \mathrm{db}$ mice. Furthermore, we also found that Enzamin treatment increased the expression of GLUT4 mRNA, which is associated with glucose uptake in muscle of $\mathrm{db} / \mathrm{db}$ mice. Previous reports suggest that the impairment of insulin signalling through the reduction of GLUT4 expression and translocation in muscle is responsible for the insulin resistance in $\mathrm{db} / \mathrm{db}$ mice ${ }^{(24,25)}$. It has been shown that TNF- $\alpha$ impairs insulin receptor signalling through the impairment of GLUT4 expression and translocation to the plasma membrane ${ }^{(26)}$. Therefore, Enzamin might improve glucose uptake in the muscle of $\mathrm{db} / \mathrm{db}$ mice, presumably through the reduction of circulating TNF- $\alpha$.

Enzamin treatment could also suppress the gene expression of the NADPH oxidase subunit, which is associated with oxidative stress, in the adipose tissue of $\mathrm{db} / \mathrm{db}$ mice. In addition to inflammation, oxidative stress plays a critical role in insulin resistance ${ }^{(27)}$. In fact, antioxidants such as vitamins $\mathrm{C}$ and $\mathrm{E}$, and $\alpha$-lipoic acid ameliorate insulin resistance ${ }^{(28,29)}$. Oxidative stress is known to be increased in obesity via $\mathrm{NADPH}$ oxidase activation ${ }^{(30,31)}$. NADPH oxidase is a major source of reactive oxygen species in various organs, especially in white adipose tissue ${ }^{(30,31)}$. NADPH oxidase consists of the membrane-associated flavocytochrome b558 family of proteins, which include Nox $2\left(\mathrm{gp} 91^{\text {phox }}\right.$ ) and $\mathrm{p} 22^{\text {phox }}$, as well as the cytosolic components $\mathrm{p} 47^{\text {phox }}, \mathrm{p} 67^{\text {phox }}$ and p $40^{\text {phox(32) }}$. In the present study, we observed that Enzamin treatment suppressed the gene expressions of these subunits. Our findings suggest that an antioxidative effect of Enzamin may also be involved in the improvement of insulin resistance.

We found that Enzamin treatment suppressed LPS-induced TNF- $\alpha$ expression in macrophages in vitro, indicating that Enzamin can directly suppress the pro-inflammatory response in macrophages. Furthermore, we observed that Enzamin treatment suppressed activated macrophage markers, such as CD68 and Toll-like receptor 4 (TLR-4), in the adipose tissue of $\mathrm{db} / \mathrm{db}$ mice. These findings suggest that Enzamin may suppress the inflammatory response in macrophages.

Morange et al. ${ }^{(33)}$ reported that PAI-1 deficiency improved insulin resistance and markedly increased tissue-type plasminogen activator (t-PA) activity, but not urokinase-type plasminogen activator (u-PA) activity in the adipose tissue of obese mice. Furthermore, the levels of plasma insulin and blood glucose in obese t-PA-deficient mice were higher than those in obese wild-type (WT) mice, suggesting that t-PA is associated with insulin sensitivity ${ }^{(34)}$. We observed that Enzamin treatment markedly decreased the levels of PAI-1 mRNA in the adipose tissue of $\mathrm{db} / \mathrm{db}$ mice. Furthermore, we previously demonstrated that Enzamin treatment increased t-PA activity, but not u-PA activity, in the blood of mice. We also reported that Enzamin contains the substances that strongly bind t-PA assessed by the IAsys resonance assay ${ }^{(9)}$. Therefore, it is assumed that the pro-fibrinolytic state induced by Enzamin might be associated with the improvement of insulin resistance in obese mice. However, further study will be required to clarify the mechanisms by which Enzamin improves insulin resistance in $\mathrm{db} / \mathrm{db}$ mice.

In conclusion, the present data suggest that Enzamin can ameliorate insulin resistance presumably through suppression of the inflammatory response and oxidative stress in adipose tissue. Since Enzamin has been utilised in health care and no side effects have been reported, it may represent a beneficial supplement for the prevention of the metabolic syndrome.

\section{Acknowledgements}

We appreciate the animal care facility at Kinki University.

There is no funding available to the present research. All issues in the present study have been planned, performed and evaluated by the authors themselves.

O. M. and Y. T. designed the research; Y. T. and M. Y. conducted the research; O. M., K. O., S. U., H. K. and Y. T. analysed the data; O. M. and Y. T. wrote the manuscript. All of the authors read and approved the final manuscript.

There are no conflicts of interest to declare.

\section{References}

1. Grundy SM, Brewer HB Jr, Cleeman JI, et al. (2004) Definition of metabolic syndrome: report of the National Heart, Lung, and Blood Institute/American Heart Association conference on scientific issues related to definition. Circulation 109, 433-438.

2. Grundy SM (2012) Pre-diabetes, metabolic syndrome, and cardiovascular risk. J Am Coll Cardiol 59, 635-643.

3. Kalupahana NS, Moustaid-Moussa N \& Claycombe KJ (2012) Immunity as a link between obesity and insulin resistance. Mol Aspects Med 33, 26-34.

4. Arsenault BJ, Pibarot P \& Despres JP (2009) The quest for the optimal assessment of global cardiovascular risk: are traditional risk factors and metabolic syndrome partners in crime? Cardiology $113,35-49$.

5. Gustafson B (2010) Adipose tissue, inflammation and atherosclerosis. J Atheroscler Thromb 17, 332-341.

6. Conde J, Scotece M, Gomez R, et al. (2011) Adipokines: biofactors from white adipose tissue. A complex hub among inflammation, metabolism, and immunity. Biofactors 37, 413-420.

7. Katagiri H, Yamada T \& Oka Y (2007) Adiposity and cardiovascular disorders: disturbance of the regulatory system consisting of humoral and neuronal signals. Circ Res 101, 27-39.

8. Mathieu P, Lemieux I \& Despres JP (2010) Obesity, inflammation, and cardiovascular risk. Clin Pharmacol Ther 87, 407-416.

9. Tamura Y, Okada K, Kawao N, et al. (2011) Profibrinolytic effect of Enzamin, an extract of metabolic products from Bacillus subtilis AK and Lactobacillus. J Thromb Thrombolysis 32, 195-200.

10. Enzamin Research Institute (2007) Method for Producing Health Nutritive Food. Issued by Japan Patent Office: P3902015 (in Japanese). Tokyo: Japan Patent Office.

11. Lubura M, Hesse D, Neumann N, et al. (2012) Non-invasive quantification of white and brown adipose tissues and liver fat content by computed tomography in mice. PLOS ONE 7, e37026.

12. Weisberg SP, McCann D, Desai M, et al. (2003) Obesity is associated with macrophage accumulation in adipose tissue. J Clin Invest $112,1796-1808$.

13. Morris DL, Singer K \& Lumeng CN (2011) Adipose tissue macrophages: phenotypic plasticity and diversity in lean and obese states. Curr Opin Clin Nutr Metab Care 14, 341-346. 
14. Suganami T, Tanimoto-Koyama K, Nishida J et al. (2007) Role of the Toll-like receptor $4 / \mathrm{NF}-\mathrm{\kappa B}$ pathway in saturated fatty acidinduced inflammatory changes in the interaction between adipocytes and macrophages. Arterioscler Thromb Vasc Biol 27, 84-91.

15. Suganami T \& Ogawa Y (2010) Adipose tissue macrophages: their role in adipose tissue remodeling. J Lenkoc Biol 88, 33-39.

16. Tamura Y, Sugimoto M, Murayama T, et al. (2008) Inhibition of CCR2 ameliorates insulin resistance and hepatic steatosis in $\mathrm{db} /$ db mice. Arterioscler Thromb Vasc Biol 28, 2195-2201.

17. Bouter B, Geary N, Langhans W, et al. (2010) Diet-genotype interactions in the early development of obesity and insulin resistance in mice with a genetic deficiency in tumor necrosis factor- $\alpha$. Metabolism 59, 1065-1073.

18. Tzanavari T, Giannogonas P \& Karalis KP (2010) TNF- $\alpha$ and obesity. Curr Dir Autoimmun 11, 145-156.

19. Klover PJ, Clementi AH \& Mooney RA (2005) Interleukin-6 depletion selectively improves hepatic insulin action in obesity. Endocrinology 146, 3417-3427.

20. Matsubara T, Mita A, Minami K, et al. (2012) PGRN is a key adipokine mediating high fat diet-induced insulin resistance and obesity through IL-6 in adipose tissue. Cell Metab 15, 38-50.

21. Maeda N, Takahashi M, Funahashi T, et al. (2001) PPAR $\gamma$ ligands increase expression and plasma concentrations of adiponectin, an adipose-derived protein. Diabetes 50, 2094-2099.

22. Zappala G \& Rechler MM (2009) IGFBP-3, hypoxia and TNF- $\alpha$ inhibit adiponectin transcription. Biochem Biophys Res Commun 382, 785-789.

23. Maeda N, Shimomura I, Kishida K, et al. (2002) Diet-induced insulin resistance in mice lacking adiponectin/ACRP30. Nat Med 8, 731-737.

24. Kato M, Suwa A \& Shimokawa T (2004) Glucose catabolic gene mRNA levels in skeletal muscle exhibit non-coordinate expression in hyperglycemic mice. Horm Metab Res 36, 513-518.
25. Yamashita Y, Okabe M, Natsume M, et al. (2012) Cacao liquor procyanidin extract improves glucose tolerance by enhancing GLUT4 translocation and glucose uptake in skeletal muscle. J Nutr Science $1,1-9$.

26. Guilherme A, Virbasius JV, Puri V, et al. (2008) Adipocyte dysfunctions linking obesity to insulin resistance and type 2 diabetes. Nat Rev Mol Cell Biol 9, 367-377.

27. Houstis N, Rosen ED \& Lander ES (2006) Reactive oxygen species have a causal role in multiple forms of insulin resistance. Nature 440, 944-948.

28. Evans JL \& Goldfine ID (2000) $\alpha$-Lipoic acid: a multifunctional antioxidant that improves insulin sensitivity in patients with type 2 diabetes. Diabetes Technol Ther 2, 401-413.

29. Ristow M, Zarse K, Oberbach A, et al. (2009) Antioxidants prevent health-promoting effects of physical exercise in humans. Proc Natl Acad Sci U S A 106, 8665-8670.

30. Furukawa S, Fujita T, Shimabukuro M, et al. (2004) Increased oxidative stress in obesity and its impact on metabolic syndrome. J Clin Invest 114, 1752-1761.

31. Park J, Chung JJ \& Kim JB (2007) New evaluations of redox regulating system in adipose tissue of obesity. Diabetes Res Clin Pract 77, Suppl. 1, S11-S16.

32. Lassegue B, San Martin A \& Griendling KK (2012) Biochemistry, physiology, and pathophysiology of NADPH oxidases in the cardiovascular system. Circ Res 110, 1364-1390.

33. Morange PE, Lijnen HR, Alessi MC, et al. (2000) Influence of PAI-1 on adipose tissue growth and metabolic parameters in a murine model of diet-induced obesity. Arterioscler Thromb Vasc Biol 20, $1150-1154$.

34. Morange PE, Bastelica D, Bonzi MF, et al. (2002) Influence of t-pA and $\mathrm{u}-\mathrm{PA}$ on adipose tissue development in a murine model of diet-induced obesity. Thromb Haemost 87, 306-310. 